Voix et Images

\title{
Charlevoix et Challe : regards sur les Anglais ou l'histoire au service d'idéologies antagonistes
}

\section{Pierre Berthiaume}

Volume 8, numéro 1, automne 1982

Madeleine Gagnon

URI : https://id.erudit.org/iderudit/200367ar

DOI : https://doi.org/10.7202/200367ar

Aller au sommaire du numéro

Éditeur(s)

Les Presses de l'Université du Québec

\section{ISSN}

0318-9201 (imprimé)

1705-933X (numérique)

Découvrir la revue

\section{Citer cet article}

Berthiaume, P. (1982). Charlevoix et Challe : regards sur les Anglais ou l'histoire au service d'idéologies antagonistes. Voix et Images, 8(1), 83-96.

https://doi.org/10.7202/200367ar d'utilisation que vous pouvez consulter en ligne. 


\title{
Charlevoix et Challe: regards sur les Anglais ou l'histoire au service d'idéologies antagonistes
}

\author{
par Pierre Berthiaume, Université d'Ottawa
}

En 1688, le capitaine Cyprian Southack s'empare de Chédabouctou en Acadie. Deux écrivains relatent l'incident: François Xavier de Charlevoix dans son Histoire et Description générale de la Nouvelle-France et Robert Challe dans son Journal d'un voyage fait aux Indes orientales, puis dans ses Mémoires. Toutefois, en confrontant les textes, le lecteur se rend vite compte que si l'issue du conflit reste la même chez les deux écrivains, la façon de rapporter les faits, en revanche, varie beaucoup et que le sens a donner à l'affrontement change radicalement d'un auteur à l'autre. Notre projet est d'illustrer comment les points de vue différents des deux écrivains renvoient en fait à des conceptions coloniales et sociales antinomiques. Précisons que le texte de Charlevoix ne nous servira que de point de comparaison pour définir ce que nous estimons être l'expression d'une idéologie chez Challe'.

Rappelons le court passage dans lequel Charlevoix évoque l'attaque anglaise des habitations de Chédabouctou:

Mais ce général ne comprenoit aparemment pas sous le nom de la Nouvelle France, ni l'Acadie, ni les Provinces circonvoisines, quoique par le Traité de Breda elles eussent été déclarées en faire partie; car tandis qu'il faisoit à $M$. de Dénonville la protestation, que je viens de dire, il envoya piller l'Habitation du Baron de St. Castin à Pentagoët, \& les Pêches sédentaires, établies à Camceaux \& à Chedabouctou. II est vrai qu'il désavoua ces entreprises; mais on avoit des preuves certaines qu'il en étoit l'Auteur, aussi-bien que des ravages, que fit bientôt après en plusieurs endroits de la Colonie un Parti de trois-cent Iroquois. En un mot toute sa conduite jusqu'à ce que la guerre fut déclarée entre les deux Couronnes, ne différa de celle, qu'il tint après cette déclaration, qu'autant que la perfidie la plus noire diffère d'une guerre ouverte. ${ }^{2}$

Même en parcourant rapidement le texte de Charlevoix, une première constatation s'impose: chez lui, le récit de la prise du fort de Chédabouctou est limité à sa plus simple expression et paraît dénué de toute substance narrative: Charlevoix ne raconte pas l'événement, mais affirme et rapporte un fait qui lui sert surtout à dénoncer la déloyauté et la malignité du gouverneur anglais: "en un mot», conclut l'historien français, «toute sa conduite jusqu’à ce que la guerre fut déclarée entre les deux couronnes ne différa de celle, 
qu'il tint après cette déclaration, qu'autant que la perfidie la plus noire diffère d'une guerre ouverte " ${ }^{3}$. Charlevoix ne relate pas la prise du fort, il ne suggère aucune péripétie du combat, il se contente de consigner le fait et préfère utiliser l'événement pour illustrer la «conduite», c'est-à-dire la mauvaise foi du gouverneur anglais qu'il condamne inexorablement. À ce sujet, du reste, ne soutient-il pas posséder «des preuves certaines» ${ }^{4}$ de l'hypocrisie du chevalier Andross qui d'une part proteste de sa volonté de «bien vivre avec» 5 le gouverneur français, le marquis de Denonville, et qui d'autre part envoie "piller" 6 les habitations françaises de Canceau et de Chédabouctou?

C'est dire que le texte de Charlevoix traduit à la fois un regard accusateur et un point de vue légaliste. Regard accusateur parce que Charlevoix souligne surtout la perfidie anglaise et qu'il traduit son jugement par l'emploi du verbe «piller» pour décrire la défaite française. Du reste, à plusieurs endroits dans les lignes qui précèdent immédiatement l'extrait que nous venons de citer, Charlevoix exonère de tout blâme les Français en choisissant d'accuser plutôt les Anglais qui arment les Iroquois, le chef huron Kondiaronk, surnommé le Rat, qui dresse si habilement les Hurons contre les Français et ceux-ci contre les premiers qu'il parvient à tromper tout le monde ${ }^{7}$, les Abénakis qui désobéissent aux ordres du gouverneur et qui montent une expédition contre les Iroquois et les Mahingans, les alliés des Anglais ${ }^{8}$, voire les "habitants», soit les Acadiens "demeurés dans la débauche ${ }^{9}$ et leurs chefs dont «l'avarice» serait directement responsable de la prise du fort par les troupes anglaises. En somme, Charlevoix accuse tout le monde sauf les Français en général et le marquis de Denonville en particulier.

De plus, le point de vue paraît légaliste dans la mesure où les premières lignes du texte insistent sur la mésinterprétation du traité de Breda qui restituait I'Acadie à la France en 1667. D'ailleurs, à deux reprises, l'auteur souligne la mauvaise foi des Anglais qui ne respectent pas leur parole et qui ont recours à la «perfidie la plus noire» 11 pour duper les Français. Charlevoix soupèse ici les gestes des Anglais sur la balance de I'honneur: à lire son texte, on ne sait pas très bien si le plus grave pour lui consiste à voir les Anglais s'emparer des habitations acadiennes ou à les surprendre en train de manquer à leur parole.

En somme, l'événement n'est mentionné qu'en passant. Ce qui importe à Charlevoix, c'est d'insérer celui-ci dans la toile historique qu'il brosse. Aussi la prise de Chédabouctou ne constitue-t-elle qu'une pièce supplémentaire à intégrer au réquisitoire contre les Anglais, jugés selon les critères de I'honneur. L'événement est vidé de sa substance et il devient un signe qui trouve place dans la logique historique de Charlevoix. Ainsi son discours, fondé sur les valeurs de l'ancien régime sert-il davantage à la défense et à l'illustration des Français qu'à la révélation du passé. Ce que cherche Charlevoix, ce n'est pas de raconter le passé, mais de démontrer l'honnêteté, I'honneur, bref la supériorité des Français catholiques sur les Anglais réformés. Son discours sépare le bon grain de l'ivraie, départage les bons des vilains à partir de valeurs finalement fondées sur l'autorité divine: ainsi, au moment de raconter la défaite anglaise devant Québec en 1690, Charlevoix 
en profite pour citer la réponse du comte de Frontenac à la sommation anglaise quand celui-ci accuse Guillaume d'Orange d'être «un Usurpateur, qui a violé les droits les plus sacrés du sang \& de la Religion»12 en détrônant Jacques II, ou les propos de Valresne qui traite l'amiral Phipps de "Corsaire» ou de "Pirate» ${ }^{13}$ sous prétexte qu'il s'est emparé de l'Acadie et qu'il a manqué à sa parole. Honneur, droit légal tiré du droit divin, voilà les assises sur lesquelles Charlevoix érige son Histoire générale de la Nouvelle-France: le récit du Jésuite ne fait que traduire par la narration des valeurs qui préexistent, qui précèdent la relation proprement dite.

Trouvons-nous le même phénomène chez Robert Challe ${ }^{14}$ Deux textes, l'un tiré des Mémoires, l'autre du Journal d'un voyage fait aux Indes orientales, et qui portent tous deux sur la prise de Chédabouctou, peuvent nous aider à définir le rôle du récit historique chez l'auteur des Illustres Françaises ${ }^{15}$.

Si l'on compare les deux textes de Challe, une première caractéristique frappe le lecteur: la relation du Journal amplifie nettement celle des Mémoires et surtout insiste davantage sur la lâcheté des officiers français. Par exemple, dans les Mémoires, on lit que «les canons n'étaient pas même montés»16, ce qui peut expliquer la facilité avec laquelle les Anglais s'emparent du fortin, alors que dans le Journal, le texte souligne la lâcheté des officiers en signalant que «le fort était bien garni de trente canons bien montés" 17 . Ou encore, si dans les Mémoires, les Anglais mettent «deux cents hommes à terre" 18 , dans le Journal, leur nombre est réduit à " un seul détachement " ${ }^{19}$. De plus, dans les Mémoires, les Anglais se cachent «le ventre à terre, à la porte du fort» ${ }^{20}$ et profitent de l'ouverture de celles-ci «à la pointe du jour» pour investir l'habitation alors que dans le Journal, Challe suggère l'insouciance des soldats français par l'emploi du participe présent: "les portes du fort étant ouvertes» 21 . Mais l'amplification des maladresses et l'insistance sur les bévues des Français se mutent en accusation quand Challe évoque la conduite de La Boulaye: pris au lit «avec une sauvage» 22 dans les Mémoires, le gouverneur est pris "dans son lit, dormant entre des filles ou femmes sauvages, sans sentinelle, \& sans tirer un seul coup de pistolet 23 dans le Journal. Honni dans les Mémoires par le ministre Seignelay qui aurait avoué à Challe «que s'il pourrait tenir La Boulaye, il le ferait pendre» 24 , le gouverneur subit une condamnation nettement plus explicite dans le Journal: Seignelay «l'a fait chercher partout», affirme Challe, «il a été condamné à être pendu» 25 .

Si le Journal et les Mémoires rapportent le même événement, le premier accentue cependant le réquisitoire contre le gouverneur et ses officiers. Dans le Journal, le récit devient l'occasion pour Challe de répandre sa bile sur La Boulaye et de laisser libre cours à sa colère contre les officiers français. D'ailleurs la fonction émotive du texte transparaît tout au long du récit. En effet, non seulement Challe insiste-t-il sur les fautes des Français, mais en plus, il signale sans cesse son ressentiment: les phrases exclamatives ${ }^{26}$, l'ironie caustique du texte 27 ou le recours à des jurons ${ }^{28}$ révèlent la rage à peine retenue de l'auteur. Du reste, Challe ne cache pas que le récit lui «rappelle un cruel ressouvenir» ${ }^{29}$ qui le porte à intervenir directement 
dans la relation et à passer des commentaires présents sur l'événement passé ${ }^{30}$. Il est vrai que la perte de ses biens l'a totalement ruiné, détail sur lequel il ne revient pas moins de cinq fois ${ }^{31}$ et qui explique sans doute sa fureur.

La fonction émotive domine dans cette relation où la présence du narrateur se fait sentir sans arrêt, où le sens même de l'anecdote se trouve moins dans le projet historiographique que dans l'expression de l'amertume de l'auteur. Mais, aussi paradoxal que cela puisse paraître, la bile de Challe ne se déverse pas sur les Bostonnais qui ont ruiné l'habitation de Chédabouctou, mais sur ceux qui étaient chargés de la défendre, sur les Français. Ainsi Challe évite même de rapporter les maux dont il a souffert lorsqu'il a été conduit à Boston ${ }^{32}$, préférant, de son propre aveu, revenir "à ce gouverneur» ${ }^{33}$ qu'il va charger de tous ses malheurs. Mieux, non seulement Challe accuse-t-il davantage les Français que les Anglais, mais en plus, c'est tout juste s'il ne perçoit pas ces derniers comme des justiciers: « $M$. de Seignelay», explique l'écrivain du roi, "voulait mettre ordre à cette mauvaise conduite des officiers français, mais les Anglais ne lui en donnèrent pas le temps. $x^{34}$ En somme l'armée anglaise devient le bras séculier de la justice. Nous sommes fort loin du texte de Charlevoix qui tranchait nettement en faveur des Français. Toutefois, ici comme chez Charlevoix, le récit sert d'exutoire à un regard bien particulier des choses, à une vision bien caractéristique du monde.

Un premier indice signale les paramètres idéologiques de Challe: si l'écrivain du roi accuse avec tant de véhémence les officiers français, c'est que ceux-ci ne remplissent pas leur mission, soit "garder Chédabouctou» 35 , mais qu'ils profitent de leurs fonctions pour faire eux-mêmes la traite: «les officiers", explique l'auteur, "au lieu de retenir leurs soldats dans leur devoir, ne les employaient qu'à traiter les pelleteries des sauvages; et eux à table, ou à la chasse toute la journée, consommaient notre poudre \& notre plomb. » ${ }^{36}$ En somme, au lieu d'appuyer et de protéger les marchands, la milice leur fait une concurrence déloyale et, qui pis est, les vole: en effet, les officiers ne consomment-ils pas les «liqueurs \& marchandises de traite ${ }^{37}$ des négociants? Comportement qui scandalise tellement Challe qu'il se surprend alors à proférer des jurons. Il est vrai que le crime lui paraît capital. En effet, si nous éclairons ce récit sur la prise de Chédabouctou par d'autres textes de l'écrivain, nous constatons rapidement que pour Challe seul l'intérêt commercial compte qui remplace même l'identité nationale. Ainsi lorsqu'il rappelle dans ses Mémoires la dureté des autorités françaises, les vexations et les impôts auxquels sont en butte les marchands de l'Acadie, Challe ajoute des remarques qui en disent long sur le rôle social qu'il prête au négoce et sur l'importance qu'il accorde à l'intérêt dans l'être humain:

Leur dureté a forcé ces gens d'entretenir un commerce secret, par la rivière Saint-Jean, avec leurs anciens correspondants. Les commandants français en sont venus jusqu'à en faire pendre quelques-uns, ce qui a révolté tous les autres, qui se sont en grande partie, retirés dans les bois, enragés de la cruauté de leur propre nation. De concert avec les sauvages, ils assommaient tous les Français 
d'Europe, qui étaient venus dans leur pays et qui osaient mettre le pied dans les bois, ou s'écarter des habitations.

Les Anglais ne les laissaient pas manquer de fusils, de poudre, ni de plomb. Ils sont tous à présent sous la même domination, et je ne doute point que quelque antipathie naturelle qui existe entre eux, par le sang et la religion, ces Français de l'Acadie n'aiment mieux obéir à des gens qui connaissent parfaitement leurs intérêts, et par conséquent les traitent avec douceur, qu'à leurs propres compatriotes, dans lesquels ils trouvent des vautours et des bourreaux. ${ }^{38}$

Du reste, ce credo, Challe l'affirmait déjà lorsque dans une lettre datée du 21 octobre 1683, il défendait les intérêts de sa compagnie dont les activités lui paraissaient entravées par l'autorité de De Meulles, l'intendant de la Nouvelle-France: "pour le commerce avec les Anglais, I'on a répondu que les habitants de l'Acadie ne recevant aucunes marchandises de France, il devait leur être permis d'en prendre des étrangers et ce d'autant plus qu'ils semblaient être tout à fait abandonnés de leurs compatriotes. 39

Si Charlevoix propose une approche légaliste de la question coloniale, pour sa part Challe choisit d'analyser le problème uniquement sous l'angle commercial ${ }^{40}$, ce qui lui permet de comprendre, sinon de légitimer le raid opéré par les Bostonnais contre Chédabouctou. En effet, Challe saisit très nettement que l'affrontement militaire entre Français et Anglais en Amérique résulte de leur concurrence commerciale. C'est pourquoi, dans les Mémoires, l'explication de l'attaque anglaise de Chédabouctou se trouve dans la volonté des Anglais d'exercer des représailles contre les Français qui ont pillé des barques bostonnaises: «j'ai dit que Bergier avait capturé en 1684 les barques anglaises, qui venaient à la pêche sur les côtes de l'Acadie. Je dirai maintenant de quelle manière les Anglais prirent leur revanche" 41 , explique Challe juste avant de narrer la prise de Chédabouctou. Ainsi le regard de Challe affirme la primauté du commerce et de l'intérêt sur les notions de légitimité, voire de nationalité. Ce que le récit de la prise de Chédabouctou révèle, c'est l'importance que Challe accorde au commerce auquel il veut subordonner les officiers de sa majesté. Pour lui, le rôle de l'armée se résume à protéger les marchands, leurs investissements et leurs biens. D'ailleurs, il est intéressant de rappeler le paragraphe qui précède immédiatement le récit de la défaite française en Acadie dans le Journal car il manifeste clairement les valeurs auxquelles se rattache Robert Challe et auxquelles le récit de la prise du fort acadien sert d'exemple antinomique:

J'ose le dire, instruit par ma malheureuse expérience: toutes les compagnies \& les colonies françaises périront, à moins que le roi n'abandonne absolument le commerce aux marchands. Les Compagnies en seront plus respectées, le commerce fleurira plus que jamais, le royaume s'enrichira davantage, \& le service en sera fait avec plus d'exactitude. ${ }^{42}$

Le regard que propose Challe paraît assez éloigné de la politique officielle de la France. Aussi on ne doit pas se surprendre de constater que lui, qui a mille raisons de détester les Anglais ${ }^{43}$, les regarde cependant comme des 
modèles à imiter car ils représentent dans son esprit des idéaux sur les plans colonial et social. Sur le plan colonial, les Anglais organisent leurs colonies exactement comme Challe souhaite voir la France développer les siennes. Autant au sujet de la conduite à tenir avec les Amérindiens ${ }^{44}$ qu'au sujet de la nécessité de promouvoir les mariages entre Blancs et Amérindiens, Challe cite l'exemple des Anglais pour formuler ce qu'il estime être la politique de peuplement à suivre: les autorités, croit-il, « devront même faciliter ces sortes d'alliances, parce que par ce moyen, ils seront instruits de tout ce qui se trame contre eux. Les Anglais ont utilement employé cette politique et l'emploient encore tous les jours." ${ }^{45}$ On le voit, l'exemple anglais lui sert de modèle pour exposer ses propres théories. Du reste, le recours au modèle anglais paraît constant dans ses Mémoires lorsqu'il agite la question coloniale. Par exemple, lorsqu'il résume ses idées sur la façon de développer la colonie, Challe se sert à la fois de l'autorité du cardinal de Richelieu et de l'exemple des Anglais: en effet, le prélat français aurait soutenu

qu'il aurait été à souhaiter que la France n'eût point entretenu de galères, parce qu'au lieu d'y mettre tant de forçats, on aurait pu les envoyer, chargés de chaînes, dans l'Acadie, qui étant regardée comme un autre monde, aurait inspiré plus de terreur que les galères; parce que ceux qu'on y envoie ont toujours l'espérance d'en revenir, au lieu qu'en les reléguant dans une contrée que le commun peuple regarde comme un pays perdu, la certitude de ne jamais revoir leur patrie aurait fait trembler ceux qui auraient voulu les imiter dans leurs crimes.

Ces malheureux, obligés de défricher la terre, auraient accompli une véritable pénitence corporelle de leurs fautes. En outre, ce travail aurait été très avantageux aux colonies et à la France: aux colonies, par la culture des terres, à la France, parce que sans bourse délier, elle aurait fondé un royaume aussi riche et florissant qu'elle était ellemême. ${ }^{46}$

Et pour appuyer davantage cette politique, spontanément l'exemple anglais apparaît dans le texte; Richelieu, au dire de Challe.

louait, à ce sujet, ce qui avait été fait par le roi d'Angleterre, Charles II, qui ne voulut pas venger la mort de son père, par l'effusion de sang d'aucuns de ceux qui y avaient contribué, et se contenta de les envoyer dans la Nouvelle Angleterre, où ces perfides emmenèrent avec eux tous ceux qui étaient attachés à leur fortune. II disait que cet acte de clémence était en même temps sage et politique, puisqu'il avait rendu ces misérables à l'État, en augmentant sa puissance. ${ }^{47}$

Plus loin, dans ses Mémoires, Challe signale la «fermeté » et la «constance 48 des Anglais qui tracent ainsi la voie aux Français. Plus explicite encore, la suite du texte montre que c'est l'ensemble de la politique coloniale anglaise qui est à suivre:

La terre existe, voilà une certitude (soutient Challe), défrichez-la, elle est à vous; défendez-vous contre vos ennemis, vous vivrez après en repos. Faites ce qu'ont fait les enfants de Jacob, pour entrer dans la terre de promission, ce que les Anglais ont fait dans la Nouvelle- 
Angleterre. Permettez-en l'entrée à tous vos compatriotes, réservez-vous le commerce extérieur, facilitez celui de l'intérieur. Ne souffrez point de bouches inutiles. N'ayez que des prêtres séculiers, d'une vie exemplaire. Punissez sévèrement le vice et la mauvaise foi, récompensez la vertu: Distinguez ceux qui ont des dispositions pour un art et leur fournissez les moyens de s'y perfectionner. Faites assembler la jeunesse à certains jours, pour l'exercer aux armes. ${ }^{49}$

Or ce qu'illustre essentiellement l'exemple anglais, c'est que l'axe sur lequel doit s'articuler toute politique coloniale est le commerce. Ainsi, au moment où il note dans son Journal que la flotte marchande des Anglais et des Hollandais mouille sous la forteresse de Madras, protégée par les canons du fort, Challe ajoute: "on appelle cela assurer son commerce: c'est qu'ils l'entendent, \& que la France ne veut pas s'en donner la peine.»50 Manifestement pour Challe, l'essentiel d'une politique coloniale se résume à laisser la plus grande marge d'autonomie aux colonies ${ }^{51}$, 'à protéger le commerce et surtout à le favoriser en en limitant le plus possible les barrières:

M. Colbert opposait que c'était une colonie naissante, dont il ne fallait troubler, ni l'ordre, ni l'économie, ni l'établissement; qu'au contraire, il fallait faciliter l'un et l'autre et suivre, en cela, l'exemple que donnait l'Angleterre qui n'exigeait rien des nouveaux établissements, afin de leur donner les moyens de s'enrichir et de multiplier; que l'entrée dans la vieille Angleterre des marchandises qui venaient de la nouvelle rapportait plus, par la douane, que n'aurait pu faire l'impôt qu'on aurait établi; qu'il en était de même entre la France et le Canada, que le commerce qui se faisait, de ce pays en France, $y$ apportait plus de profit que l'impôt n'en pourrait produire, outre qu'une infinité d'ouvriers gagnaient leur vie dans la fabrication des chapeaux de castor, qu'on n'était plus obligé d'aller chercher en Moscovie et qu'il en était ainsi des martres et des loutres.

II ajoutait que le commerce qui se pratiquait entre les Français d'Europe et les Occidentaux, était également profitable aux uns et aux autres, en ce que ceux-ci n'ayant de commerce qu'avec I'ancienne France et y apportant tout ce que le pays produit, telles que sont les pelleteries et la morue, et retirant de l'ancienne France tout ce qui leur est nécessaire: bas, souliers, linge, draps, vins, eaux-de-vie, poudre, plomb, fusils, en un mot, tout ce qui leur faut, tant pour leur usage personnel que pour la traite avec les sauvages, le Roi gagnait à la fois sur l'entrée dans le royaume, des pelleteries et du poisson et sur la sortie de ce qu'ils emportaient, si bien que le tout montait bien plus haut que le tribut qu'ils s'étaient imposé.

Il faisait ressortir encore que ce tribut était volontaire de leur part, mais qu'il en irait autrement, sitôt qu'il serait levé par des fermiers, au nom du Roi, que même il rapporterait beaucoup moins, parce que les enfants du pays accoutumés à fréquenter les sauvages, et qu'à cause de cela, on nomme coureurs des bois, s'imaginant que cet impôt n'était levé que pour enrichir des gens d'affaires, et non pour être employé à la défense ou à l'embellissement du pays, bien loin d'apporter leurs pelleteries à Québec, les porteraient à Orange, aux 
Anglais, desquels ils retireraient, en échange, des toiles, des draps, de la quildive, ou eau-de-vie de sucre, ce qui causerait un très grand tort aux manufactures du royaume et aux distillateurs d'eaux-de-vie, sans compter qu'il pourrait rester dans les esprits des ferments de division, susceptibles de porter de mauvais fruits dans l'avenir. 52

Challe est si conscient des effets néfastes des impôts sur le commerce qu'il n'hésite pas à conspuer même les plus petites taxes. Et ici encore, l'anecdote historique lui permet d'illustrer et de démontrer sa thèse: en effet, au moment où il évoque la question du tribut perçu sur les produits commerciaux par les autorités civiles ou religieuses et qu'il en dénonce les effets désastreux, Challe rapporte l'exemple d'une taxe perçue sur le produit de la pêche aux moules: au moment où, constate l'auteur, l'abbesse de l'abbaye royale de la Grâce Notre-Dame de Charron obtient le privilège de lever un impôt de "deux liards ou six deniers de droit sur (les) moules, par charge d'enfant, un sol pour la charge d'un homme ou d'une femme, un sol et six deniers pour la charge d'un âne et deux sols pour la charge d'un cheval» 53 , la mer semble se tarir soudainement. L'abbesse abandonne-t-elle son impôt, «le lendemain de sa renonciation, les moules reparurent plus épaisses et plus nombreuses qu'elles n'avaient jamais été.» ${ }^{54}$ L'histoire, ici anecdotique, est embrigadée et mise au service d'une démonstration. Finalement l'histoire sert de preuve à une thèse et paraît enrôlée dans une idéologie, dans une idéologie caractérisée essentiellement par la primauté du commerce surtout au sein de la société.

Aussi Challe en vient-il à définir le rôle du souverain en fonction du commerce, voire à subordonner l'autorité royale au négoce: «en effet», explique-t-il, «les Hollandais reconnaissent, encore aujourd'hui, que leurs ancêtres et eux-mêmes ne doivent l'extension de leur commerce par tout le monde, et par conséquent leurs richesses, qu'à leur industrie encouragée, par un prince qui entendait bien ses propres intérêts, en facilitant le leur». ${ }^{5}$ En somme, si Jean le Bon, comte de Flandre, est excellent prince, c'est qu'il a su reconnaître son intérêt dans ceux de ses sujets et qu'il a su faire coïncider son pouvoir avec les nécessités du commerce: On mesure la distance qui existe entre la pensée de Challe et la politique pratiquée par la France. Le moins qu'on puisse dire, c'est que la conception challienne de l'autorité royale se distngue nettement de celle que professe la France monarchique. D'ailleurs Challe lui-même est conscient de la différence de rapports qui existent entre le souverain et le peuple dans une monarchie parlementaire, de type anglais, et dans une monarchie absolutiste: ainsi, pour Challe,

les Français idolâtrent leur Roi, à proprement parler, ils consentent d'en être les esclaves plutôt que les enfants, ils ne se plaignent que des ministres et jamais de leur souverain; ils imputent tout le bien à celui-ci et tout le mal aux autres. II n'en est pas de même chez les Anglais; ils aiment leur Roi, tant qu'il n'attaque pas leur religion ni leurs privilèges et qu'il ne s'écarte pas du serment qu'il jure à son sacre et à son couronnement. Mais, sitôt qu'il viole l'un ou l'autre, ils se tiennent quittes du leur. ${ }^{56}$ 
On comprend dès lors que le recours au modèle anglais permet à Challe d'avancer des propositions sans trop se compromettre. En effet, la prudence avec laquelle Challe dénonce les autorités françaises paraît remarquable: presque toujours Challe prête à quelqu'un d'autre les propos qu'il tient contre les autorités françaises ou les critiques qu'il formule. Par exemple, il cite le discours de la reine Anne sur l'Acadie pour attaquer le traité d'Utrecht ${ }^{57}$, les propos du cardinal Richelieu pour s'opposer à l'envoi de délinquants aux galères plutôt qu'en Acadie ${ }^{58}$, ceux de Colbert lorsqu'il s'élève contre les taxes et les redevances imposées par la métropole à ses colonies ${ }^{59}$, ou enfin ceux de Denizy, un interprète, lorsqu'il s'en prend aux Jésuites ${ }^{60}$. Nous pourrions multiplier à l'infini les exemples qui montrent que pour dénoncer les autorités françaises ou leur politique, Challe reprend les propos d'autrui, à moins qu'il en prête à quelqu'un, et par là se sert d'une personne pour avancer et pour appuyer ses propositions. Ainsi le modèle anglais lui sert doublement: d'une part il lui permet d'exposer ses propres thèses en les fondant sur un exemple, ce qui produit une preuve de leur possibilité, de l'autre, il lui permet d'afficher ses idées sans s'impliquer trop ouvertement.

Aussi l'histoire, et plus précisément l'histoire coloniale ${ }^{61}$, joue-t-elle un rôle important chez Challe qui utilise l'anecdote historique pour appuyer et pour fonder ses thèses. Exactement comme dans le cas des personnages derrière lesquels Challe se dissimule pour exposer ses principes coloniaux et sociaux en rappelant leurs propos ou en leur en prêtant, il utilise l'histoire et la fait parler et la revêt d'un message précis: I'histoire assume alors doublement les idées de l'auteur qui se dérobe derrière l'autorité des faits: elle parle et elle expose des thèses à la place de Challe et en plus, elle explicite ces thèses en en devenant elle-même l'exemple, la preuve de leur possibilité et leur caution. En somme, I'histoire énonce une vision sociale précise en plus de l'illustrer. Chez Challe, I'histoire actualise une idée, une thèse, une idéologie en la montrant, en la rendant perceptible par le biais du récit.

Mais s'agit-il bien d'histoire ici, c'est-à-dire de récit qui veut témoigner d'un événement passé. On l'a déjà constaté, sans cesse Challe utilise les Anglais pour définir son propre projet, ou plutôt son propre idéal colonial. Mais bien sûr, prêter aux Anglais des intentions uniquement fondées sur le mercantilisme ou voir leur politique comme un tout cohérent, c'est prêter à la politique anglaise et à l'histoire coloniale britannique des intentions, des buts, des méthodes qu'elles n'avaient pas: c'est y voir plus qu'elles ne contenaient. Par exemple, les intentions évangélisatrices sont loin d'être absentes des préoccupations des colons britanniques, comme en témoignent les entreprises d'un George Berkeley au XVIIle siècle. Ainsi ce que fait Challe, c'est de projeter sur les Anglais ses propres intentions, ses propres visées coloniales et il ne retient de l'expérience anglaise que ce qui lui convient. Aussi l'histoire coloniale est-elle utilisée et devient-elle une création, presque une fiction, inventée par un écrivain en mal de fournir des modèles, des exemples pour appuyer, pour fonder et pour exposer ses thèses. Sous la plume de Challe, l'expérience coloniale anglaise se métamorphose en canon qui sert à soutenir la vision de l'auteur. Les Anglais sont utilisés comme étalon. Aussi est-il normal dans la 
logique challienne qu'ils apparaissent dans le récit de la prise de Chédabouctou pour punir les Français: le modèle vient corriger la réalité.

L’histoire permet donc de concrétiser une idéologie. Aussi il n'est pas étonnant que les récits de Charlevoix et de Challe sur la prise de Chédabouctou soient si différents: tous deux témoignent de conceptions radicalement antinomiques sur la société et sur la politique coloniale française. Autant Charlevoix paraît tourné vers le passé et semble s'accrocher à la notion de monarchie absolue de droit divin, autant Robert Challe annonce la montée d'une bourgeoisie qui devra attendre la révolution de 1789 pour s'emparer du pouvoir. Aussi I'histoire, ici d'un épiphénomène, somme toute assez secondaire, des rivalités qui opposent la France et l'Angleterre en Amérique, devient-elle le champ dans lequel s'expriment des idéologies. La relation $n$ 'a plus rien d'objectif et elle paraît tributaire de conceptions sociales, de visions du monde qui la précèdent et qui l'ordonnent. Le récit historique n'est plus que l'espace dans lequel se réalisent les visions du monde de ceux qui croient rapporter et éclairer le passé. L'histoire n'éclaire que le présent, que le présent de ceux qui l'écrivent. L'anecdote, ici en principe véridique, sert de support à une idéologie. Le récit possède ainsi une nouvelle fonction: créer un passé qui témoigne du présent, des espoirs et des déceptions des hommes qui, écrivant l'histoire du passé, vivent une histoire contemporaine.

1. Précisons avec Louis Althusser que l'idéologie constitue le «système des idées, des représentations qui domine l'esprit d'un homme ou d'un groupe social" («Idéologie et appareil idéologique d'État», La Pensée, 151, Paris, juin 1970, p. 22). Plus explicite, Denis Monière précise que l'idéologie «est un système global plus ou moins rigoureux de concepts, d'images, de mythes, de représentations qui dans une société donnée affirme une hiérarchie de valeurs et vise à modeler les comportements individuels et collectifs. Ce système d'idées est lié sociologiquement à un groupe économique, politique, ethnique ou autre, exprimant et justifiant les intérêts plus ou moins conscients de ce groupe». (Le Développement des idélogies au Québec, Montréal, Québec/Amérique, 1977, p. 13).

2. Charlevoix, François-Xavier de. Histoire et description générale de la NouvelleFrance avec le journal historique d'un voyage fait par ordre du roi dans l'Amérique septentrionale, Paris, Nyon, 1744, tome I, p. 538. La protestation à laquelle Charlevoix fait allusion porte sur l'affirmation du gouverneur Andross de "bien vivre» (op. cit., p. 538) avec les Français.

3. Ibid.

4. Ibid.

5. lbid.

6. Ibid.

7. Charlevoix, op. cit., I, pp. 535-538.

8. Charlevoix, op. cit., I, p. 535.

9. Charlevoix, op. cit., I, p. $\mathbf{5 4 1 .}$

10. Charlevoix, op. cit., I, p. 540.

11. Charlevoix, op. cit., I, p. 538.

12. Charlevoix, op. cit., II, p. 80 .

13. Ibid.

14. Bien entendu on s'attend à ce que le texte de Challe soit plus étoffé puisque l'auteur rapporte un épisode de sa propre vie. Cependant, ce n'est pas tant la 
quantité de faits narrés qui nous importe que la façon de voir ceux-ci. Ajoutons que Charlevoix accorde assez d'importance à l'habitation de Chédabouctou pour demander à $\mathrm{N}$. Bellin de dresser un plan détaillé de celle-ci. En effet, le «plan de la Baye de Chédabouctou aujourd'hui Havre de Milfort", qui se trouve au tome II entre les pages 420 et 421 , présente une carte très précise de Chédabouctou: Bellin y indique la profondeur de l'eau, l'emplacement des bancs de sable, le relief les accidents géographiques et il situe le "village des Sauvages», le «bourg» ainsi que le «fort".

15. Faute de posséder une édition exacte des Mémoires, nous n'utiliserons ce texte que pour éclairer le Journal d'un voyage fait aux indes orientales (1690-1691) bien que le récit du Journal, mis au net vraisemblablement en 1708 (voir la dédicace du Journal d'un voyage fait aux Indes orientales (1690-1691). Paris, Mercure de France, 1979, p. 57) précède celui des Mémoires dont le premier cahier a été achevé en 1716 (voir la préface de Frédéric Deloffre et Mélâhat Menememcioglu du Journal, p. 32). Toutefois, comme le soulignent Frédéric Deloffre et Mélâhat Menememcioglu, certains passages du Journal ont été écrits plus tard (voir note 6, p. 560 et note 837, p. 629 du Journal) et surtout, le Journal est "non seulement la première œuvre de Challe, mais la plus personnelle, celle où il apparaît, lui I'homme de la sincérité, sous la figure la moins apprêtée qu'on puisse concevoir.» (Frédéric Deloffre, Mélâhat Menememcioglou, préface op. cit., p. 35). De là notre préférence du récit du Journal à celui des Mémoires.

16. Challe, Robert, Mémoires, Paris, Plon, 1931, p. 272. A l'avenir, nous désignerons cette ceuvre par le mot Mémoires.

17. Challe, Robert, Journal d'un voyage fait aux Indes orientales (1690-1691), texte intégral, établi avec introduction et notes par frédéric Deloffre et Mélâhat Menememcioglou, Paris, Mercure de France, 1979, p. 215. A l'avenir, nous désignerons cette œuvre par le mot Journal.

18. Mémoires, p. 272.

19. Journal, p. 215. "Detachement, s. m. Choix qu'on fait de quelques gens de guerre dans plusieurs Compagnies, ou Regiments, pour les envoyer à quelque expédition ou attaque. Le Mareschal de Camp commandoit un detachement de l'armée de six mille hommes. On le dit même des corps qui font partie d'une armée. On fit un detachement de dix soldats de chaque Compagnie." (Antoine Furetière, Dictionnaire universel, La Haye \& Rotterdam, Arnout \& Reinier Leers, 1690. Est-il besoin de préciser que les exemples proposés sont inversés par rapport aux deux sens du mot?)

20. Mémoires, p. 272.

21. Journal, p. 215.

22. Mémoires, p. 272.

23. Journal, p. 215.

24. Mémoires, p. 272.

25. Journal, p. 216.

26. Par exemple «mais nos liqueurs \& marchandises de traite, qu'ils se faisaient donner de force!» (Journal, p. 215), "beau spectacle pour moi!» (Journal, p. 215) ou enfin «deux femmes à cet âge!» (Journal, p. 215).

27. En effet Challe signale ironiquement que le fortin * fut pourtant, grâce à la vigilante conduite du gouverneur \& des officiers, pris" (Journal, p. 215) sans combat, ou encore, après avoir dénoncé l'incurie de la Boulaye, Challe ajoute que ales autres officiers, sages imitateurs d'une si judicieuse conduite, furent tous pris comme lui» (Journal, p. 215).

28. "encore disaient-ils en vivant à discrétion comme dans une ville prise d'assaut, qu'ils étaient bien malheureux de servir des b... de marchands qui étaient auprès de leur feu à se gratter les c... avec leurs maîtresses." (Journal, p. 215).

29. Journal, p. 215. 
30. Par exemple, "passe pour celui-ci (Journal, p. 215) ou encore, "deux femmes à cet âge! était-ce pour le réchauffer en plein été? ou, avait-il le diable dans les reins? J'ignore où il s'est retiré.» (Journal, p. 215).

31. "Je perdis tout ce que je possédais au monde", affirme Challe, ou encore, «ils m'avaient pris, moi, avec une barque chargée de pour plus de cinquante mille écus de castors", ou, «je les vis arriver à Boston, où j'étais, avec pour plus de cinquante mille francs de pelleteries", ou "y ayant été ruiné de fond en comble", ou enfin, "y aurais-je rattrapé mon bien?" (Journal, p. 215 et 216).

32. "Ill n'est pas question ici de dire comment je fus traité», précise-t-il à ce sujet. (Journal, p. 215).

33. Journal, p. 215.

34. p. 215.

34. Journal, p. 215.

35. Journal, p. 215.

36. Journal, p. 215.

37. Journal, p. 215.

38. Mémoires, p. 275.

39. Challe, Robert, lettre datée du 21 octobre 1683, publiée dans la Revue d'Histoire littéraire de la France, 78 e année, $n^{\circ} 6$, Paris, Armand Colin, novembre-décembre 1979 , p. 1007. Signalons qu'une abondante correspondance fait foi des problèmes soulevés par cette question du commerce des Acadiens avec les colons anglais établis à Boston. Par exemple, dans un «mémoire sur l'Acadie», daté du 1er décembre 1687, De Meneval affirme être «informé que le dit Sr Perrot a faict-charger l'année dernière plus de 80 barriques d'eau de vie avec des vins et des toilles qu'il a faict porter à Baston ". (Collection des manuscrits contenant lettres, mémoires, et autres documents historiques relatifs à la Nouvelle-France, recueillis aux archives de la province de Québec, ou copiés à l'étranger, Québec, A. Côté et Cie, 1883, volume I, p. 410.) Une lettre du roi, datée du 21 février 1688 , le montre "surpris que le Sr Perrot continue de faire avec les Anglois un commerce deffendu " (op. cit., l, p. 415). Enfin, une lettre du ministre à Perrot, datée du 21 janvier 1688, lui intime l'ordre d'arrêter de détourner les produits envoyés en Acadie sur Boston (op. cit., I, p. 416).

40. Par exemple, contrairement à Charlevoix, la question de la légitimité de Guillaume d'Orange paraît à Challe un problème sans importance: «Que m'importe à moi \& aux autres commerçants que leur roi s'appelle Jacques ou Guillaume? " Journal, p. 112). Du reste, Challe signalera encore à deux reprises dans son Journal le ridicule de cette question de légitimité (voyez le Journal aux pages 282 et 520 ) et il en parlera aussi dans ses Mémoires (Mémoires, p. 188).

41. Mémoires, pp. 272-272.

42. Journal, p. 214.

43. En effet, plus qu'un autre, Challe a toutes les raisons de détester les Anglais: ceux-ci l'ont ruiné en s'emparant du fort de Chédabouctou, de plus ils représentent une menace constante pour la colonie de la Nouvelle-France: "ceux qui, comme moi», explique-t-il, "ont été dans l'Acadie et le Canada et qui connaissent la pêche de la morue, la fertilité du terroir, l'étendue du pays, qui ont pratiqué la traite avec les sauvages et savent la facilité que les Anglais auront à nous boucher le fleuve du Saint-Laurent: ceux-là, dis-je savent aussi qu'il aurait été plus avantageux à la France de leur céder la Normandie, la Bretagne et même l'Aquitaine, comme ils l'ont eue autrefois, que de leur céder l'Acadie, Terre-Neuve et la baie d'Hudson." (Mémoires, p. 24). Plus loin, il revient sur le sujet et il soutient "que le traité d'Utrecht coûtera bien du sang, ou que la Nouvelte-France fera bientôt partie de la NouvelleAngleterre." (Mémoires, p. 92). D'autre part, sur le plan humain, Challe méprise la lâcheté qu'il décèle chez les Anglais: au combat de La Hougue, il constate: Il faut pourtant dire à leur honte, qu'ils n'osaient pas, seul à seul, affronter un vaisseau français, qui en avaient au moins toujours deux ou trois contre lui.» (Mémoires, pp. 176-177). De même, il déteste leur cruauté et il note qu'aux Antilles, "les Anglais y ont fait des cruautés plutôt dignes de démons que 
d'hommes» (Journal, p. 519). Du reste, pour Challe les Anglais constituent une «nation cruelle, tigresse \&traîtresse» (Journal, p. 482).

44. Challe ne parle-t-il pas dans son Journa/de la «bonté ridicule dont les seuls Français sont capables"? (Journal, p. 349).

45. Mémoires, p. 259.

46. Mémoires, pp. 77-78.

47. Mémoires, p. 78.

48. Mémoires, p. 242.

49. Mémoires, pp. 242-243.

50. Journal, p. 306. Signalons que Challe cite aussi très souvent dans son Journal l'exemple donné par les Hollandais qui entendent bien protéger leur commerce et leurs colonies: voir Journal, pages 214, 216-217, 279 et 374.

51. Par exemple, alors qu'il note que la colonie est abandonnée par la France, Challe insiste pour signaler que celle-ci a pu se débrouiller pour survivre et pour assumer sa propre défense: «lls ne tiraient, comme je l'ai dit, aucun secours de la France européenne. Cependant, tout cela ne s'était pas fait et ne pouvait pas s'entretenir sans défense. Pour y subvenir, ils s'étaient imposé à eux-mêmes un droit sur les pelleteries, qui montait au quart des castors. Outre cela, ils s'étaient volontairement imposé un droit d'entrée sur les vins, eaux-de-vie, tabacs et autres marchandises sèches. Ce droit était fort, le premier qui le perçut et le fit valoir fut un nommé Bazire, dont j'ai encore vu la femme vivante, en 1683. II était honnête homme, et de concert avec le Conseil de Québec, mit toutes choses en ordre et en état de défense.» (Mémoires, p. 80). Ensuite Challe regrette que les autorités aient mal réagi à ces initiatives: "Il se trouva des gens, qui ne jugèrent pas bon que ceux de Québec se fussent mis, d'eux-mêmes, dans un état tranquille. On fit entendre au Roi, qu'il n'y avait que lui qui devait porter l'épée dans son royaume et dans les lieux qui relevaient de la couronne, que les habitants de la NouvelleFrance avaient usurpé ses droits et blessé son autorité, que c'était même d'une dangereuse conséquence pour la suite, parce que ces gens, accoutumés à ne dépendre que d'eux-mêmes, ne voudraient plus reconnaître le pouvoir souverain, lorsqu'on voudrait les y assujettir, et se mettraient insensiblement sur le pied de ne reconnaître la France européenne, que comme les Anglais, leurs voisins, reconnaissent la vieille Angleterre, seulement pour et par le pavillon.» (Mémoires, pp. 80-811.

52. Mémoires, pp. 81-82. Cet axe importe tant à Challe qu'il y subordonne tous ses jugements sur les affaires coloniales. Par exemple, s'il s'oppose à la venue de frères mendiants dans la colonie, c'est pour éviter qu'il y ait des bouches inutiles à nourrir et pour empêcher les religieux de soutirer de l'argent aux colons, argent qui ne servirait plus dès lors au commerce: «J'ai dit qu'il n'y faut mener, ni souffrir aucune bouche inutile. Je le répète encore. Les moines de quelque ordre qu'ils soient, sont, comme dit M. du Bellay, des cruches, qui ne se baissent que pour se remplir et qui, peu à peu, s'emparent de tout le bien d'un État, sans en porter les charges.» (Mémoires, p. 244). De même, s'il insiste pour punir la mauvaise foi, c'est qu'elle ruine la confiance entre les gens et par là, qu'elle nuit au commerce: "La probité et la droiture régnaient autrefois en France», constate Challe, "et à présent il n'y en a plus. Les Français étaient autrefois renommés pour leur bonne foi, ils sont à présent regardés d'un autre œil. Cette vertu qui est le premier et le plus puissant lien de la société civile s'est perdue par degrés, à mesure que leurs chefs leur en ont montré l'exemple." (Mémoires, p. 31). Et dans le Journal, on trouve des remarques qui vont dans le même sens: «Ce n'est pas seulement le commerce de la Hollande qui a abattu le nôtre: c'est nous-mêmes qui y avons le plus contribué, \& y contribuons encore le plus par l'indulgence que les juges ont pour les banqueroutiers, auxquels, aux dépens d'un honneur que ces scélérats ont foulé aux pieds, la justice en France conserve la vie." (Journal, p. 368).

53. Mémoires, p. 132.

54. Mémoires, p. 132.

55. Mémoires, p. 77. Du reste, le critère challien par excellence pour juger les mesures politiques ou sociales prises par les autorités se confond avec l'idée de commerce. 
Challe jauge-t-il Colbert ou Pontchartrain, il le fait sous l'angle du commerce: "Ce commerce", constate-t-il, "si florissant sous le ministère de M. Colbert, s'est tout à fait anéanti depuis que Pontchartrain lui a succédé." (Mémoires, p. 4). Challe veut-il résumer sa pensée sur la révocation de l'édit de Nantes, il le fait à partir d'un point de vue mercantile: "La suppression de l'édit de Nantes a commencé la perte de la France, par l'argent que ceux qui s'en sont bannis ont emporté avec eux, et par les manufactures qui faisaient une partie du commerce du royaume, que ces bannis volontaires ont porté chez nos voisins." (Mémoires, p. 4). De même ce qu'il reproche essentiellement au traité d'Utrecht, c'est d'avoir entériné la perte de l'Acadie, de Terre-Neuve et de la baie d'Hudson dont I'Angleterre a bien percu l'importance commerciale et stratégique (voir les Mémoires aux pages 25-26).

56. Mémoires, p. 23.

57. Mémoires, p. 25.

58. Mémoires, pp. 77-78.

59. Mémoires, pp. 81-82.

60. Mémoires, pp. 94-95.

61. Sans doute parce qu'il a été directement impliqué dans une entreprise coloniale qui a avorté, la politique coloniale française devient pour Challe le lieu privilégié pour dénoncer la pensée des autorités françaises en matière de colonisation. Du reste, Challe se démarque très nettement de la politique officielle de la France, de cette politique dont témoigne, par exemple, un Charlevoix. Ainsi, pour lui, si la France veut concurrencer l'Angleterre dans le domaine colonial, elle ne doit pas tant miser sur l'armée ou sur la conversion des Amérindiens, à laquelle Challe ne fait pratiquement jamais allusion sinon pour dénoncer les Jésuites, que sur le négoce. Ainsi, si on veut faire cesser le commerce entre les Acadiens et les colons de la Nouvelle-Angleterre, il suffit de leur procurer tout ce qui leur est nécessaire et surtout de «leur vendre à meilleur prix que les Anglais" (Mémoires, p. 275). En ce sens, Challe paraît bien être cet "homme de la moyenne bourgeoisie" que décrit Roger Francillon dans un article. (Robert Challe: I'authenticité du "Journal de voyage aux Indes orientales", Revue d'Histoire fittéraire de la France, $79 e$ année, $n^{\circ} 6$, Paris, Armand Colin, novembre-décembre 1979, p. 943.) 